\title{
All-Optical Format Conversion From NRZ to BPSK Using a Single Saturated SOA
}

\author{
Cishuo Yan, Yikai Su, Member, IEEE, Lilin Yi, Student Member, IEEE, Lufeng Leng, Member, IEEE, \\ Xiangqing Tian, Student Member, IEEE, Xinyu Xu, and Yue Tian
}

\begin{abstract}
All-optical format conversion from nonreturn-to-zero (NRZ) to binary phase-shift keying format is experimentally demonstrated at $8 \mathrm{~Gb} / \mathrm{s}$ using a single semiconductor optical amplifier (SOA). The conversion is based on the gain and phase modulations of the SOA on an input NRZ signal of finite extinction ratio.
\end{abstract}

Index Terms-Amplitude-shift keying (ASK), format conversion, phase-shift keying (PSK), semiconductor optical amplifier (SOA).

\section{INTRODUCTION}

A LL-OPTICAL format conversion has received much interest recently since all-optical signal processing is considered as one of the key aspects of future optical networks. There have been a number of reports on format conversion schemes [1]-[4]. However, most of them are between on-off keying signals, such as nonreturn-to-zero (NRZ), return-to-zero (RZ), and carrier-suppressed return-to-zero. While amplitude-shift keying (ASK) formats are widely employed in optical systems and networks of all scales, phase-shift keying (PSK) formats have been demonstrated advantageous over conventional ASK signals in long-haul transmission systems [5], [6]. As PSK may become the chosen format for future long-distance communications, format conversion from ASK to PSK would be desirable at an intermediate node between a metro network and a long-haul backbone (Fig. 1). However, few demonstrations have been performed on conversions from ASK to PSK formats. In [7], four semiconductor optical amplifiers (SOAs) in a Mach-Zehnder configuration with two assisting-light signals were used to achieve cross-phase modulation for ASK to binary phase-shift keying (BPSK) conversion. In this process, wavelength conversion is inevitable, which may not always be desired.

In this letter, we present a simple yet effective method to convert NRZ signals to PSK format by employing a single saturated SOA. We show that the input ASK format data with a finite extinction ratio (ER) can be converted to PSK format with

Manuscript received June 26, 2006; revised September 12, 2006. This work was supported by the NSFC (60407008), by the 863 program, by the key project of Ministry of Education (106071), and by the Shanghai Rising Star Program (04QMX1413).

C. Yan, Y. Su, L. Yi, X. Tian, X. Xu, and Y. Tian are with the State Key Laboratory of Advanced Optical Communication Systems and Networks, Department of Electronic Engineering, Shanghai Jiao Tong University, Shanghai 200240, China (e-mail: yikaisu@sjtu.edu.cn).

L. Leng is with New York City College of Technology, City University of New York, Brooklyn, NY 11201 USA.

Color versions of Figs. 3-6 are available online at http://ieeexplore.ieee.org. Digital Object Identifier 10.1109/LPT.2006.885633

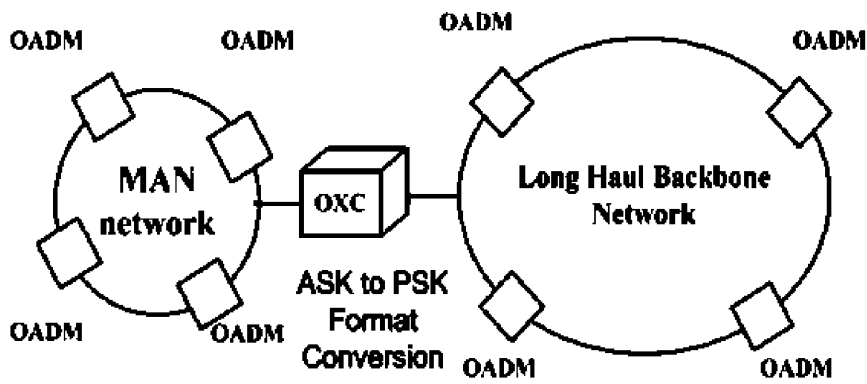

Fig. 1. ASK-to-PSK conversion is desired between a metro network and a longhaul backbone.

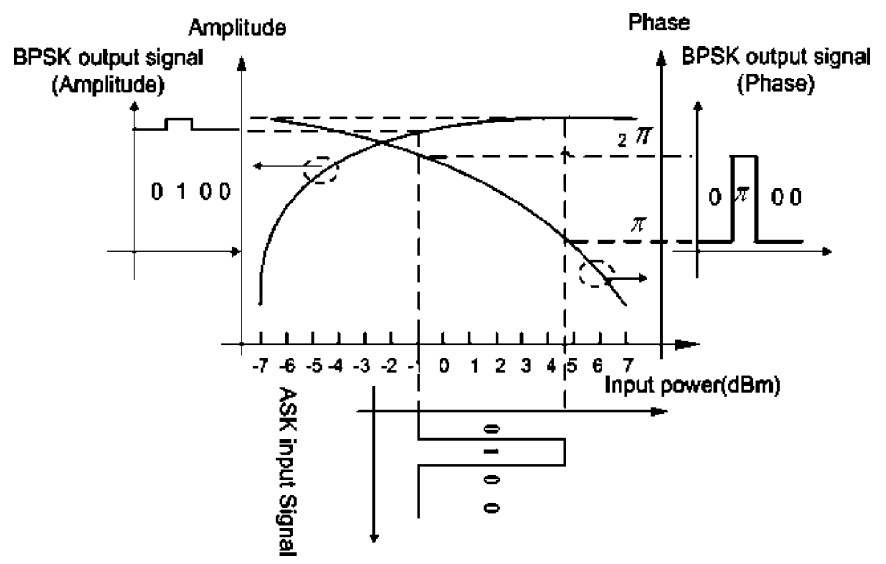

Fig. 2. "1"s and "0"s have different input powers that result in different phase and amplitude modulation.

almost no power penalty. It was discussed in [8] that input signals with different powers experience different gains and varied phase shifts due to the nonlinear properties of the SOA. In this experiment, we take advantage of the combined self-phase modulation (SPM) and gain-saturation effects of the SOA to realize NRZ-to-PSK conversion without wavelength change. This SOA-based format-conversion scheme is advantageous due to the small size, low cost, and easy integration features.

Fig. 2 illustrates the principle of operation. When the SOA is operated in the saturation regime, the " 0 " level of the input signal is more amplified than the " 1 " level [8]. At the output, although their amplitudes are close, the phases can be very different. By properly adjusting the input power, an ASK signal with a finite ER can be converted to BPSK format exhibiting approximately constant amplitude and a phase difference of $\pi$ between " 0 "s and " 1 "s. Note that the bit rate in this configuration is mainly limited by the recovery time of the SOA, which should be less than one bit duration. 

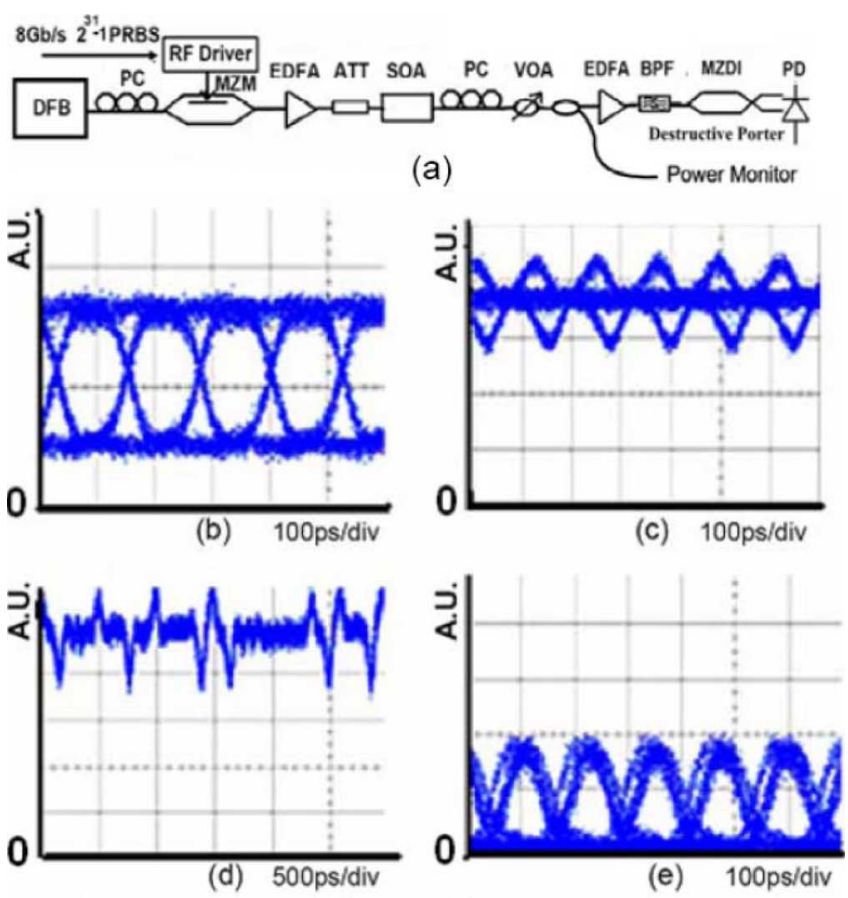

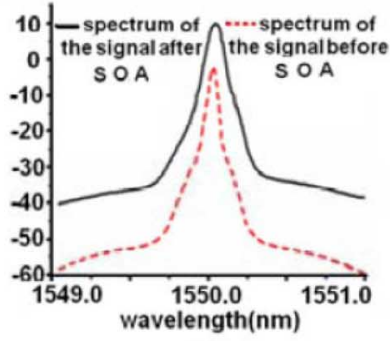

(f)

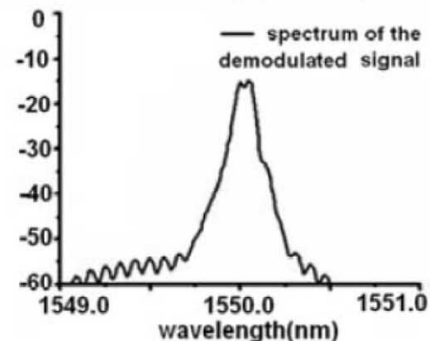

(g)
Fig. 3. (a) Experimental setup. PC: Polarization controller. ATT: Attenuator. BPF: Bandpass filter. VOA: Variable optical amplifier. PD: Photodetector. (b) Eye diagram of the input signal to the SOA with an ER of $5.5 \mathrm{~dB}$. (c) Eye diagram of the output signal from the SOA. (d) Output signal of the SOA. (e) Eye diagram of the demodulated signal from the destructive port of the MZDI. (f) Optical spectrum of tlie input NRZ signal (dashed line) and the converted BPSK signal (solid line). (g) Optical spectrum of the demodulated signal.

\section{EXPERIMENTAL SETUP AND RESULTS}

In this experiment, the SOA is a commercial polarization-maintaining booster amplifier (model SOA-NL-OEC-1550 from CIP) with a gain recovery time of approximately $45 \mathrm{ps}$. This SOA has a small signal gain of $30 \mathrm{~dB}$ and a saturation power of $10 \mathrm{dBm}$. The driving current is set to $250 \mathrm{~mA}$. The experimental setup is shown in Fig. 3(a). The NRZ signal is generated by a Mach-Zehnder modulator driven with an $8-\mathrm{Gb} / \mathrm{s}$ pseudorandom bit sequence (PRBS) of length $2^{31}-1$, and the corresponding eye diagram is provided in Fig. 3(b). The data rate is determined by the delay length of the available Mach-Zehnder delay interferometer (MZDI), which is used as the data decoder. An erbium-doped fiber amplifier (EDFA) amplifies the signal to $10 \mathrm{dBm}$, and an attenuator is employed to decrease the power to $2 \mathrm{dBm}$, which reaches the deep saturation region of the SOA. After the SOA, " 1 "s and "0"s are almost at the same level due to different gains they have experienced [Fig. 3(c) and (d)] [9], [10]. The overshoot/undershoot and the chirp of the output signal are caused by the limited

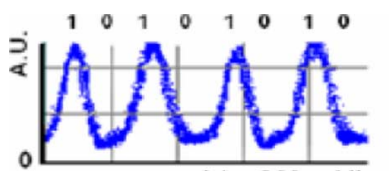

(a) $200 \mathrm{ps} / \mathrm{div}$

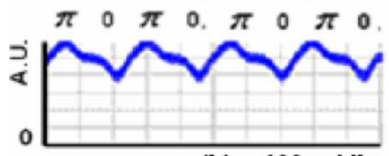

(b) $100 \mathrm{ps} / \mathrm{div}$

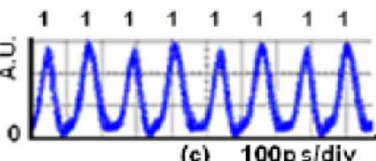

(c) 100p s/div
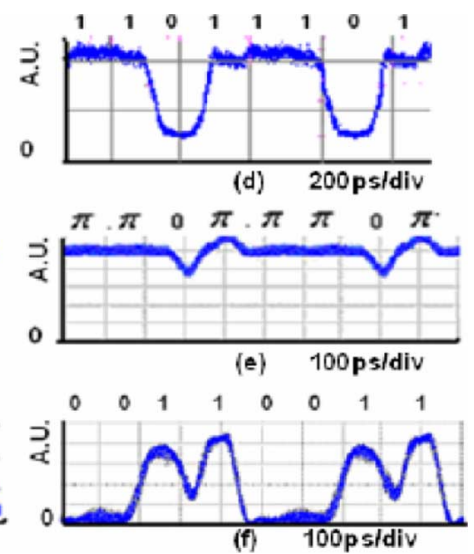

Fig. 4. Demonstration of some typical patterns. (a) Input NRZ data: 10101010. (b) Output from the SOA displays a phase modulation of $\pi 0 \pi 0 \pi 0 \pi 0$. (c) Demodulated signal: 11111111. (d) Input NRZ data: 11011101. (e) Output from the SOA experiences a phase modulation of $\pi \pi 0 \pi \pi \pi 0 \pi$. (f) Demodulated signal: 00110011.

recovery time of the SOA and mainly exist at the beginning of the pulses, which could be filtered by the MZDI. The SPM effects associated with the residual amplitude fluctuations on the PSK signal are not expected to result in significant signal degradation due to the filtering of the MZDI. In addition, an optical equalization scheme for PSK signal regeneration can be employed to suppress the amplitude fluctuation while retaining the phase information [11]. The optical signal is preamplified by an EDFA and then filtered by a tunable bandpass filter with a bandwidth of $1.6 \mathrm{~nm}$. Then, the signal is demodulated by an MZDI with 1-bit delay. We observe that an RZ-like format signal rather than an NRZ signal is obtained at the destructive port of the MZDI. This is mainly caused by the chirps associated with the overshoot/undershoot. The chirp of the PSK signal is also dependent on the recovery time of the SOA [12]. For the particular SOA used in this experiment, if the ER of the input signal is adjusted to $5.5 \mathrm{~dB}$, the phase difference between " 1 "s and " 0 "s of the output signal is $\pi$. When further increasing the ER of the input signal, the phase difference deviates from $\pi$. The phase information is quantified based on the amplitude of the demodulated signal, and the highest amplitude corresponds to the $\pi$ phase shift.

To further prove the effectiveness of our scheme, we use some typical data patterns to serve as the input data and observe the output at the destructive port of the MZDI. Fig. 4(a) shows the input NRZ data pattern "10101010", Fig. 4(b) presents the signal output from the SOA, and Fig. 4(c) provides the waveforms of the demodulated signal at the destructive port of the MZDI. A precoding section in this particular experiment is not necessary due to the properties of PRBS. Note that the results also apply to NRZ-to-DPSK conversion. In practice, a postdecoding stage would be employed to ensure the correct reception of the data. Fig. 4(d)-(f) illustrate another typical pattern "11011101". We also note that duobinary and alternate mark inversion formats are obtained at the constructive and destructive ports of the MZDI [13], respectively.

The ER of the input NRZ signal plays an important role in determining the quality of the output signal from the SOA. Fig. 5 shows the BER curves of the converted PSK signals from input signals of different ERs. It can be seen that for an ER of $5.5 \mathrm{~dB}$, 


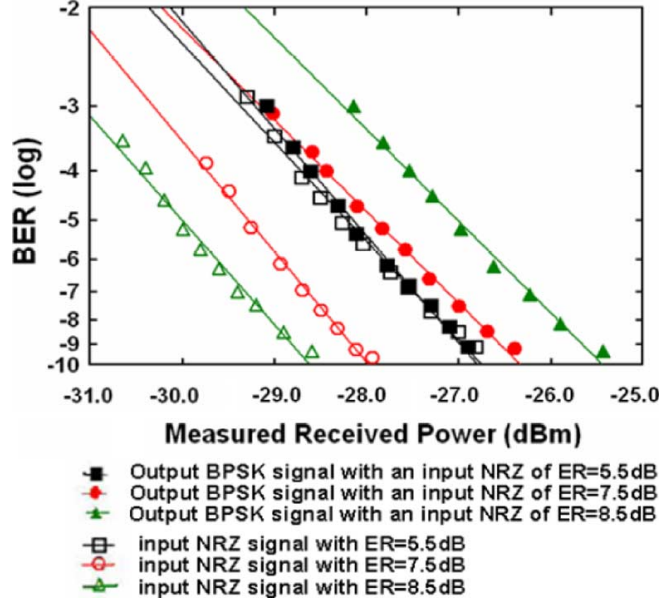

Fig. 5. BER curves of three signals of different ERs.

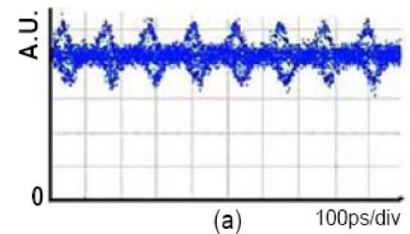

(a)

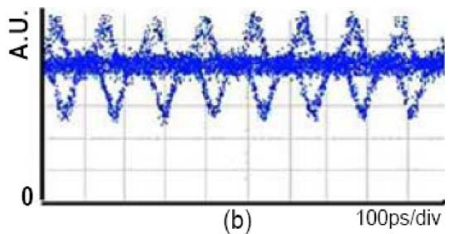

(b) the input signal can be intentionally degraded in the transmitter within a reasonable range.

\section{CONCLUSION}

We have proposed a novel and simple format conversion scheme using a single saturated SOA. We experimentally demonstrate error-free conversion from ASK to PSK at $8 \mathrm{~Gb} / \mathrm{s}$ with almost no sensitivity loss for an input signal of $\mathrm{ER}=5.5 \mathrm{~dB}$. Format conversions for input signals with higher ERs are also investigated. High data rates can be envisioned by improving the recovery time of the SOA.

\section{REFERENCES}

[1] C. G. Lee, Y. J. Kim, C. S. Park, H. J. Lee, and C.-S. Park, "Experimental demonstration of $10-\mathrm{Gb} / \mathrm{s}$ data format conversion between NRZ and RZ using SOA-loop-mirror," J. Lightw. Technol., vol. 23, no. 2, pp. 834-841, Feb. 2005

[2] J. Yu, G. K. Chang, J. Barry, and Y. Su, "40 Gbit/s signal format conversion from NRZ to RZ using a Mach-Zehnder delay interferometer," Opt. Commun., vol. 248, pp. 419-422, Apr. 15, 2005.

[3] L. Xu, B. C. Wang, V. Baby, I. Glesk, and P. R. Prucnal, “All-optical data format conversion between RZ and NRZ based on a Mach-Zehnder interferometric wavelength converter," IEEE Photon. Technol. Lett., vol. 15, no. 2, pp. 308-310, Feb. 2003.

[4] W. Li, M. Chen, Y. Dong, and S. Xie, "All-optical format conversion from NRZ to CSRZ and between RZ and CSRZ using SOA-based fiber loop mirror," IEEE Photon. Technol. Lett., vol. 16, no. 1, pp. 203-205, Jan. 2004.

[5] A. H. Gnauck, G. Raybon, S. Chandrasekhar, J. Leuthold, C. Doerr, L. Stulz, and E. Burrows, " $25 \times 40-\mathrm{Gb} / \mathrm{s}$ copolarized DPSK transmission over $12 \times 100-\mathrm{km}$ NZDF with $50-\mathrm{GHz}$ channel spacing," IEEE Photon. Technol. Lett., vol. 15, no. 3, pp. 467-469, Mar. 2003.

(b) $\mathrm{ER}=7.5 \mathrm{~dB}$

the receiver sensitivity for the converted signal is approximately the same as that of the input NRZ signal. No significant change in the receiver sensitivity was observed when the input power to the SOA was varied from 0 to $2 \mathrm{dBm}$. Because the slope of the phase curve is not a constant, one can properly adjust the power for different ERs of the input NRZ signal. A variable optical amplifier may be employed to adjust the input power in practice. Since a lower input signal power induces more amplitude fluctuation, there is a tradeoff between the amplitude fluctuation and achieving an exact differential phase of $\pi$. In the experiment, an input power of $1 \mathrm{dBm}$ was used and a phase difference of $1.3 \pi$ was measured when the ER is increased to $7.5 \mathrm{~dB}$. Although this phase-modulated signal can still be demodulated by the MZDI, signal quality degradation is observed. This is due to the fact that when bit " 1 " and " 0 " show a phase difference of $1.3 \pi$, the output amplitude at the MZDI destructive port is $\cos (0)-\cos (1.3 \pi)=1.31$, compared to 2 in the case of $\pi$ phase shift. In this case, the power penalty becomes $\sim 1.5 \mathrm{~dB}$. The degradation is also attributed to the larger chirp of the signal with higher ERs, which causes a longer recovery time in the SOA, as shown in Fig. 6.

In practice, an ER range of 8-10 dB is typically seen. In this case, optimization of the SOA to adapt to the higher ERs is needed. For instance, changing the value of the linewidth enhancement factor of the SOA so that the slope of the phase curve shown in Fig. 2 can be reduced [14]. Alternatively, the ER of
[6] T. Mizuochi, K. Ishida, T. Kobayashi, J. Abe, K. Kinjo, K. Motoshima, and K. Kasahara, "A comparative study of DPSK and OOK WDM transmission over transoceanic distances and their performance degradations due to nonlinear phase noise," J. Lightw. Technol., vol. 21, no. 9, pp. 1933-1943, Sep. 2003.

[7] K. Mishina, A. Maruta, S. Mitani, T. Miyahara, K. Ishida, K. Shimizu, T. Hatta, K. Motoshima, and K. Kitayama, "All-optical format conversion from NRZ-OOK to RZ-BPSK using SOA-MZI wavelength converter," in Proc. OFC, Anaheim, CA, 2006, Paper OThB2.

[8] T. Durhuus, B. Mikkelsen, C. Joergensen, S. Lykke Danielsen, and K. E. Stubkjaer, "All-optical wavelength conversion by semiconductor optical amplifiers," J. Lightw. Technol., vol. 14, no. 6, pp. 942-954, Jun. 1996.

[9] X. Wei, Y. Su, X. Liu, J. Leuthold, and S. Chandrasekhar, "10-Gb/s RZ-DPSK transmitter using a saturated SOA as a power booster and limiting amplifier,' IEEE Photon. Technol. Lett., vol. 16, no. 6, pp. 1582-1584, Jun. 2004.

[10] X. Liu, X. Wei, Y. Su, J. Leuthold, Y. Kao, I. Kang, and R. Giles, "Transmission, of an ASK-labeled RZ-DPSK signal and label erasure using a saturated SOA," IEEE Photon. Technol. Lett., vol. 16, no. 6, pp. 1594-1596, Jun. 2004.

[11] A. Striegler and B. Schmauss, "All-optical DPSK signal regeneration based on cross-phase modulation," IEEE Photon. Technol. Lett., vol. 16, no. 4, pp. 1083-1085, Apr. 2004.

[12] R. Inohara, K. Nishimura, M. Tsurusawa, and M. Usami, "Experimental analysis of cross-phase modulation and cross-gain modulation in SOA-injecting CW assist light," IEEE Photon. Technol. Lett., vol. 15, no. 9, pp. 1192-1194, Sep. 2003.

[13] D. Penninckx et al., "Optical differential phase shift keying (DPSK) direct detection considered as a duobinary signal," in Proc. ECOC, Amsterdam, The Netherlands, 2001, Paper We.P40.

[14] N. Pleros, G. T. Kanellos, C. Bintjas, A. Hatziefremidis, and H. Avramopoulos, "Optical power limiter using a saturated SOA-based interferometric switch," IEEE Photon. Technol. Lett., vol. 16, no. 10, pp. 2350-2352, Oct. 2004. 